\title{
Sharp Feature Detection in Point Clouds
}

\author{
Christopher Weber ${ }^{1}$, Stefanie Hahmann ${ }^{2}$, Hans Hagen ${ }^{1}$ \\ ${ }^{1}$ TU Kaiserslautern, Germany \\ ${ }^{2}$ Université de Grenoble, Laboratoire Jean Kuntzmann, France
}

\begin{abstract}
This paper presents a new technique for detecting sharp features on point-sampled geometry. Sharp features of different nature and possessing angles varying from obtuse to acute can be identified without any user interaction. The algorithm works directly on the point cloud, no surface reconstruction is needed. Given an unstructured point cloud, our method first computes a Gauss map clustering on local neighborhoods in order to discard all points which are unlikely to belong to a sharp feature. As usual, a global sensitivity parameter is used in this stage. In a second stage, the remaining feature candidates undergo a more precise iterative selection process. Central to our method is the automatic computation of an adaptive sensitivity parameter, increasing significantly the reliability and making the identification more robust in the presence of obtuse and acute angles. The algorithm is fast and does not depend on the sampling resolution, since it is based on a local neighbor graph computation.
\end{abstract}

Keywords-unstructured point sets, feature detection; sharp features; Gauss map clustering

\section{INTRODUCTION}

Over the last years scanning technologies have become more and more affordable and accurate, thus increasing their use in manufacturing, art and design.

The usage of scanning devices covers a wide area of applications.

During the development process of a new product they can be used to approve and optimize the production process or to digitize manually designed prototypes. During production they might be used to support the quality control. For example, in the car manufacturing industries the wear of a production tool can be measured by controlling the position of some fix points on the product. Today the number of control points used is relatively small and only covers some critical sections of the product.

Sharp features typically belong to those critical sections. It would thus be useful to know the points belonging to a sharp feature. With nowadays fast and precise scanning devices which provide a dense set of surface points, quality analysis of mechanical parts could therefore be improved by using a sharp feature extraction algorithm.

The detection of sharp features in a dense point cloud is important not only for quality measurements, but also for reverse engineering with mesh generation, surface reconstruction, simplification or segmentation where sharp features have to be preserved. In nonphotorealistic rendering features can enhance the visual perception. In semantic driven applications such as semantic classification or annotation of 3D models the knowledge of sharp features is a useful information.

The term "feature" has different meanings depending on the discipline and the application. In CAD "design by features" and "feature recognition" are well defined terms. Feature design consists of introducing functional or manufacturing features directly into the CAD model in order to make the design process more efficient. Feature recognition tries to extract manufacturing or form features from a solid model [JPR00]. In modeling and computer graphics the term feature is used for free form features of 3D shapes. A free form feature is a visually prominent characteristic of the shape, including salient edges, ridge and valley lines, but also sharp features as line-type or corner features.

In this paper, we present a new method for extracting sharp features on point-sampled surfaces. A pointsampled surface is a simple unstructured point cloud, where the points belong to a 2-manifold, without any further information about (mesh) connectivity, topology, parameterization or differential properties.

Our algorithm is based on the observation that sharp features have the particularity to separate clearly two or more surface parts with tangent discontinuity, in a local area around the sharp feature. We thus introduce Gauss map clustering as detection operator.

Most existing feature extraction algorithms for general free-form features use global sensitivity parameters to decide whether a point belongs to a feature or not. Sharp features however can have varying angles from obtuse to acute. We will show that a global threshold does not work well and propose instead a method to automatically adapt the threshold value.

The present method does not need any prior surface reconstruction. It works directly on the point cloud from which a local neighbor graph is computed. The method is therefore fast and independent of the sampling resolution.

The paper is organized as follows. After discussing the related works in Section 2 we present our method for sharp feature detection based on a Gauss map clustering in Section 3. In Section 4 an extension to the method is presented. The paper closes with many results shown in Section 5 and some concluding remarks about future 
research.

\section{RELATED WORKS}

There are multiple existing techniques for feature extraction. Most of them rely on polygonal meshes [HG01], [WB01], [KBSS01], [HPW05], [WG09]. These techniques are different to our case, since we work with simple unstructured point cloud data. A problem of point based methods is the lack of any normal and connectivity information of the model. This makes feature detection a more challenging task than in mesh based methods.

Several surface reconstruction methods aim to preserve sharp features when constructing a mesh from an unorganized point cloud. [AGJ00], [ACK01] reconstruct creases, corners and sharp features in a post processing step. Attene et al. [AFRS05] go one step further. They not only detect sharp features but propose a method for restoring sharp features in triangle meshes. Starting from the observation that sharp features of the original model are often smoothed-out in triangle meshes, they propose a two-step algorithm. Triangles near a sharp feature are first selected. Then subdivision of these triangles followed by a repositioning of the new vertices onto sharp features reconstructs the sharp features and thus repairs finally the triangle mesh. Our aim is however to work directly on the point cloud not with triangle meshes.

[GM97] extract surfaces, feature lines and feature junctions by discretizing the space into a volume grid and by computing then surface votes from the data points for the cells. Saliency functions based on an eigenvalue analysis of the votes and the use of an adapted marching cubes algorithm allows the computation of the features. Our goal is to efficiently handle sampled points and thus avoid volume discretization and surface reconstruction via marching cubes.

There are also MLS-surface reconstruction methods that are seeking for sharp feature reconstruction. MLS methods generally produce smooth surfaces, which makes them particularly appropriate to handle noisy point sets. Sharp feature require an extra treatment.

Fleischmann et al. [FCOS05] use robust statistics to identify sharp features. Neighborhoods of points are segmented into regions corresponding to the same surface part. To this end forward-search techniques are used to identify outliers not belonging to the local surface region. Piecewise MLS surfaces are then constructed preserving sharp features. An extension of this work by Daniels et al. [DOHS08] extract feature curves on the reconstructed MLS surface. The advantage is that points lying on the reconstructed sharp feature can be identified, even in the case of very noisy and rough input data. These points however don't necessarily belong to the input points set. Our approach is different in the sense that we seek to detect sharp feature points in the point cloud without any local surface reconstruction. However, it might be interesting to investigate if the forward search techniques used here can be adapted to directly identify feature points in point clouds.

Öztireli et al. [OGG09] use kernel regression to extend the moving least squares surface reconstruction with sharp features. Their method increases the presentation of sharp features conserving the $C^{2}$-continuity of the MLS-surface. In some applications however it would be better to have a real sharp feature with a $C^{0}$. continuous surface.

Very few methods are dedicated to point-sampled geometry only. Gumhold et al. [GWM01] present a method that uses the Riemannian tree to build the connectivity information in the point cloud. They then analyze the neighborhood of each point via a principal component analysis (PCA). The eigenvalues of the correlation matrix are used to determine a probability of a point belonging to a feature, and the kind of feature. This method can differentiate between line-type features, border and corner points. The result is a quite dense set of points covering the feature independent if the feature is sharp or not, since points with high curvature values are detected. This set of points is then reduced by computing a minimal spanning tree followed by some branch cutting. This is an elegant way to get a sparse set of points representing the feature line. It might however be seen as an disadvantage that no difference between sharp features and others can be made. Our algorithm will focus specifically to the detection of sharp features only. Indeed, points with high curvature value are not necessarily a sharp feature point. It will be shown that PCA-based methods are less appropriate in this case.

Pauly et al. [PKG03] use a related approach and extend it with a multiscaling analysis of the neighborhoods. Based on the eigenvalue analysis of the covariance matrix they compute a value for the surface variation in this local area. For the multiscaling, they vary the size of the neighborhoods to receive more information.

The multiscaling approach enhances the feature recognition, especially in noisy datasets, but increases computational cost, since the method needs to analyze up to 200 neighborhood sizes for each point in the dataset. It is not specialized to sharp features, but all kinds of other visually eminent features. Is it possible to adapt the different parameters in order to detect sharp features? The method works with an upper and an lower bound for selecting feature points, which have to be chosen very close to identify a sharp feature. But this can run into problems in case of sharp features with varying angle. We will discuss this problem of varying angles and propose a solution that works well with any kind of sharp angle.

Demarsin et al. [DVVR07] also searched for sharp features in point cloud data. But in contrast to our work, they are interested in closed sharp features. They use segmentation to identify the regions of sharp features. The output is a set of points with many points representing the feature line, which has to be sparsened. Therefore 
they use a graph approach with a minimum spanning tree, similar to Gumhold and Pauly to extract the closed feature lines.

Merigot et al. [MOG09] estimate principal curvatures and normal directions of the underlying surface from a point cloud using a so-called Voronoï covariance measure and provide valuable theoretical guarantees. A convolved covariance matrix is computed of a union of Voronoï cells and returns principal curvatures and principal directions. It can be applied to feature detection in discrete data via a proposed algorithm which iteratively computes covariance matrices with varying neighborhoods. Similar to other PCA-based algorithms, the estimated features form a large band of points near the feature line.

\section{Feature extraction}

This section presents our basic feature extraction method. An extension is given in Section 4. It consists of three steps: We first build the data structure for our points set. In the second step we create local neighborhoods in the point set. In the third step we analyze these neighborhoods to identify sharp features. Let us first define point cloud and sharp feature.

A point cloud is a simple set of 3D point coordinates $P=\left\{\boldsymbol{p}_{1}, \boldsymbol{p}_{2}, \ldots, \boldsymbol{p}_{N}\right\}, \boldsymbol{p}_{i} \in \mathbb{R}^{3}$ without any normal or connectivity information. The data points are unstructured, but supposed to belong to a 2-manifold surface. Let $N=|P|$ be the cardinal of the point set.

A sharp feature we want to detect in the point cloud can be of different nature. It can be an edge or line between two surfaces or a corner where three or more surfaces meet.

\subsection{Data structure}

To detect the sharp features in this point cloud, we are going to check first every point in the cloud for being part of a sharp feature or not. Similar to [PKG03],[GWM01], we analyze the neighborhood of each single point, to decide, if it belongs to a sharp feature or not. As neighborhood we use the k-nearest.

Space partitioning algorithms such as a kd-tree [CLR01] have good performance

as well as approximate nearest neighbor search such as Best Bin First [BL97] and Balanced BoxDecomposition Tree based search [AMN*98].

We choose a kd-tree implementation for it's simplicity. It is a pre-processing step in our algorithm, which is performed once. It has worst case complexity of $O\left(3 N^{\frac{2}{3}}\right)$, and an average complexity of $O(\log N)$ [LW77].

The result can be stored in a Riemannian graph. In this graph, the minimum spanning tree connecting the point is expanded to connect each point with its k-nearest neighbors. Hoppe et al. [HDD*92] used it in a graph optimization problem in order to compute consistently oriented normals to a point cloud for surface reconstruction. This is a quite expensive step towards surface reconstruction we don't intend to undertake here.

\subsection{Neighborhood analysis}

Having the local neighborhood $N_{p}$ of the $k$-nearest points constructed for a point $\boldsymbol{p} \in P$, we now want to analyze it to decide if the sample point belongs to a sharp feature or not. To this end we apply a Gauss map clustering.

\subsection{Discrete Gauss map}

Let $N_{p}$ be the neighborhood of $\boldsymbol{p}$ containing the $k$ nearest neighbors, and $I_{p}$ the index set of $N_{p}$. Let $T$ be the set of all possible $k \cdot(k-1)$ triangulations of $\boldsymbol{p}$ with its neighborhood points

$$
T=\left\{\quad \Delta_{i j}=\Delta\left(\boldsymbol{p}, \boldsymbol{p}_{i}, \boldsymbol{p}_{j}\right) \quad \mid \quad i \neq j, \quad i, j \in I_{p}\right\} .
$$

The normal vector of the triangle $\Delta_{i j}$ is given by

$$
\boldsymbol{n}_{i j}=\overline{\boldsymbol{p} \boldsymbol{p}_{i}} \times \overline{\boldsymbol{p} \boldsymbol{p}_{j}} .
$$

Note that $\boldsymbol{n}_{i j}=-\boldsymbol{n}_{j i}$.

The discrete Gauss map of the neighborhood of $\boldsymbol{p}$ can now be defined as the mapping of $T$ onto the unit sphere $S^{2}$ centered at $\boldsymbol{p}$ as follows

$$
\begin{aligned}
\boldsymbol{G}_{p} & : T \rightarrow S^{2} \\
\Delta_{i j} & \mapsto \boldsymbol{x}_{i j}:=\boldsymbol{p}+\frac{\boldsymbol{n}_{i j}}{\left\|\boldsymbol{n}_{i j}\right\|} .
\end{aligned}
$$

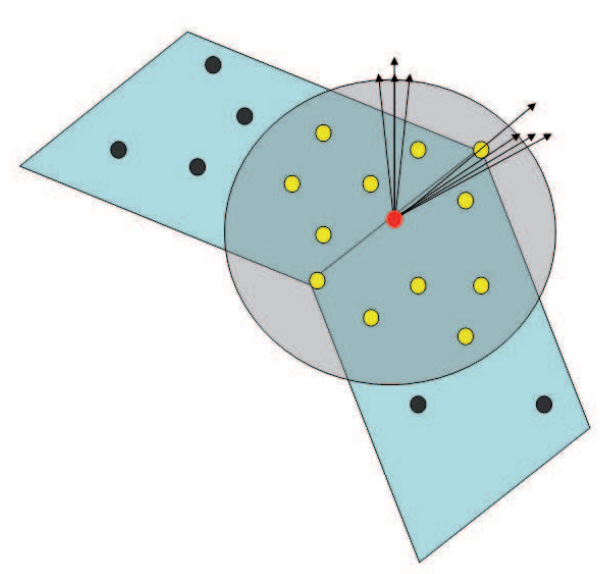

Fig. 1. Computation of the normal vectors used for feature identification in a local neighborhood.

Feature detection is now performed in two steps. A first step discards all points belonging to a planar region with a simple flatness test. The remaining feature candidate points undergo then in a second step a more precise selection process, called Gauss map clustering. Gauss map clustering can also be used for other applications, such as segmenting a point sampled geometry into connected regions grouping together points with same local curvature behavior [LX08].

\section{FLATNESS TEST}

These normal vectors allow now to draw some conclusions about the local surface behavior at $\boldsymbol{p}$ of the point cloud. If the lines passing through $\boldsymbol{p}$ and spanned by $\boldsymbol{n}_{i j}$ 
with $i<j$ are all almost parallel, then the underlying surface is nearly flat at $\boldsymbol{p}$. Note that $\boldsymbol{n}_{i j}$ and $\boldsymbol{n}_{j i}$ span the same line. To compare them, we compute the angle between these lines.

The flatness test consists now in computing the standard deviation of these angles, i.e. we check the distance that means the angle to the median value of the directions. If it is lower than a given threshold (we worked with $15 \%$, which corresponds to an angle of about 13) the surface in this neighborhood is assumed to be flat or nearly flat without having a sharp feature. But the inverse is not true. A high deviation at $\boldsymbol{p}$ does not imply a sharp feature. It can also appear in a high curvature region without a sharp feature.

\section{GAUSS MAP CLUSTERING}

That's why we also analyze $\boldsymbol{p}$ by computing the Gauss map $G_{p}$ of the set $T=\left\{\Delta\left(\boldsymbol{p}, \boldsymbol{p}_{i}, \boldsymbol{p}_{j}\right)\right\}$. Here we now check their clustering behavior.

This idea is motivated by the fact that in the case of a smooth piecewise $C^{0}$ surface the Gauss map of the neighborhood of a surface point is different whether the point is flat, curved (elliptic, hyperbolic or parabolic) or tangent plane discontinuous. In case of a nearly flat point the Gauss map of neighbor points will represent one cluster of points on the sphere. In the case of a curved point (parabolic, hyperbolic, or elliptic) the points on the sphere will be spread over a larger region. And in the case of a tangent plane discontinuity, the points of the sphere will build two distinct clusters, see Figure 2 for some typical 2D examples.
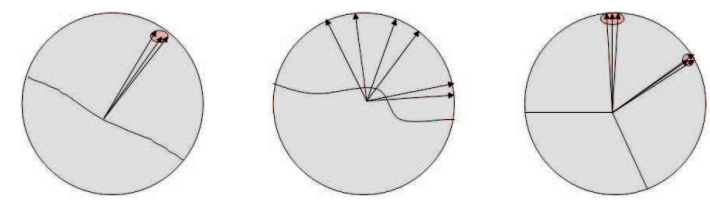

Fig. 2. 2D examples for cases during feature detection

In the case of a point-sampled surface, the difficulty is that we don't know anything about the surface nearby. We don't have a local triangulation and nor a normal vector associated to the neighbor points. For that reason we defined our Gauss map as the projection of the normals of all possible local triangulations, see Sect. 3-3. The resulting Gauss map of a sharp feature point will thus contain some additional "noisy" points which correspond to the triangles, which finally don't belong to the underlying surface. These noisy points however don't affect the general clustering behavior. In practice they will disappear when computing the clusters.

Let us illustrate these assumptions for the common case of two intersecting planes with $\boldsymbol{p}$ lying on the sharp edge. Half of the $k$ neighborhood points are lying on each plane, see Figure 3 left. Computing the Gauss map will result in two (opposite) clusters of $O\left(k^{2} / 4\right)$ identical points corresponding to the triangles lying entirely in one plane, and two clusters for the other plane. Note that $\boldsymbol{n}_{i j}$ and $\boldsymbol{n}_{j i}$ belong to opposite clusters. All other points on the sphere (noisy points) correspond to triangle where $\boldsymbol{p}_{i}$ belongs to one plane and $\boldsymbol{p}_{j}$ belongs to the other plane. These points are sparsely distributed over the sphere. In Figure 3 we show a real example implemented with Matlab. On the left, the planes and the 16 neighborhood points are shown. On the right, the Gauss map is shown, where the thick red and blue points represent the clusters.

Similar clustering behavior can be observed for the non-exhaustive list of sharp features whose profiles are shown in Figure 4.
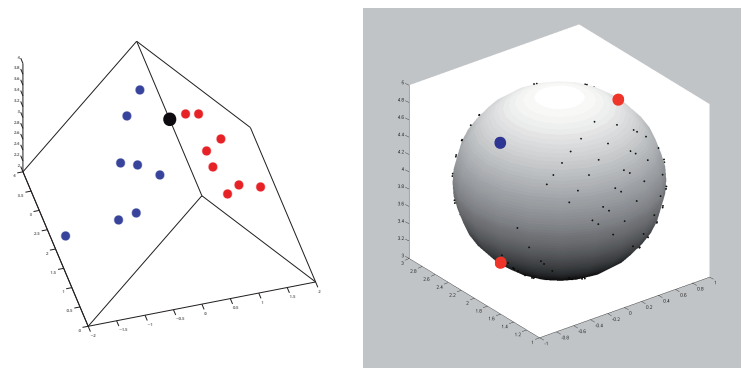

Fig. 3. Computation of the Gauss map $G_{p}$ for feature identification in a local neighborhood.

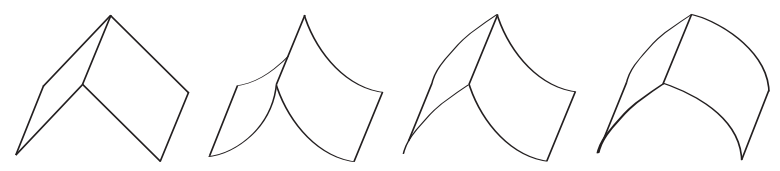

Fig. 4. Some example of sharp feature profiles.

We therefore use the clustering behavior of the projected points on the sphere, called Gauss map clustering, in order to determine whether the sample point $\boldsymbol{p}$ belongs to a sharp feature or not.

Regarding the clusters, we have to keep in mind that we can not be sure about the direction of our normals as mentioned above. That means we don't have any information whether the normals points outside or inside of the surface. But for the identification of a sharp feature this does not matter at all. We use the fact, that each point $\boldsymbol{x}_{i j}$ on the Gauss sphere has it's counterpart $\boldsymbol{x}_{j i}$ on the opposite side of the sphere, since $\boldsymbol{n}_{i j}=-\boldsymbol{n}_{j i}$. The clustering behavior of one set of points is thus reproduced identically on the other hemisphere.

Distance measure. An important step in a clustering algorithm is to choose a distance measure, which decides how close two elements are. The present point set has two particularities: it is a spherical point set and it consists of pairs of symmetric points, i.e. points lying on opposite positions on the sphere. An appropriate distance measure has to take care of this. In order to preserve symmetry in the point set we choose as distance measure the angle between the lines through 

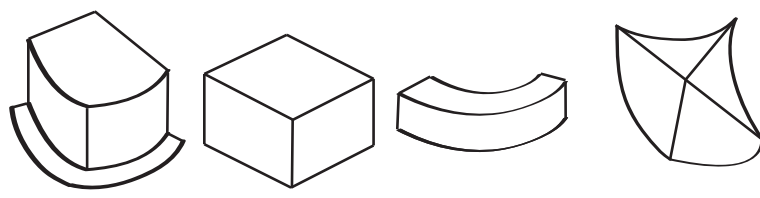

Fig. 5. Examples of sharp corners of valence three and four.

pairs of symmetric points. It can be shown that the angle between two normal vectors of our Gauss map is equivalent to the geodesic distance between two points on the sphere. The minimal angle between two lines spanned by the normal vectors is thus an appropriate distance measure satisfying the particular requirements mentioned before:

$$
d\left(\boldsymbol{x}_{i j}, \boldsymbol{x}_{j k}\right)=\min \left\{d_{g}\left(\boldsymbol{x}_{i j}, \boldsymbol{x}_{k l}\right), d_{g}\left(\boldsymbol{x}_{i j}, \boldsymbol{x}_{l k}\right)\right\},
$$

where $i, j, k, l \in I_{p}$ and

$$
d_{g}\left(\boldsymbol{x}_{i j}, \boldsymbol{x}_{k l}\right)=\arccos \left(<\boldsymbol{n}_{i j}, \boldsymbol{n}_{k l}>\right)
$$

is the geodesic distance between two points $\boldsymbol{x}_{i j}$ and $\boldsymbol{x}_{k l}$ on the unit Gauss sphere.

Clustering. Many clustering algorithms require an a priori specification of the number of clusters to produce. Our aim however is not to cluster the total point set, but to distinguish some clusters from other sparsely distributed points in the Gauss map. We therefore use a hierarchical agglomerative ("bottom-up") clustering method [HTF09]. It begins with each point as a separate cluster and merges them successively into larger clusters. As linkage criterion, which defines the distance between two clusters, we use the mean distance $D_{c}$ between elements of each cluster

$$
D_{c}\left(S_{1}, S_{2}\right)=\frac{1}{\left|S_{1}\right| \cdot\left|S_{2}\right|} \sum_{\boldsymbol{x} \in S_{1}} \sum_{\boldsymbol{y} \in S_{2}} d(\boldsymbol{x}, \boldsymbol{y}),
$$

where $S_{1}, S_{2}$ are two clusters to be compared and $d$ the distance measure of the Gauss map defined in (3). This is one of the most common criterion [HTF09]. Each agglomeration increases the distance between clusters by merging the closest clusters. To find the two closest clusters we have to compute the distances between all clusters. After the merging of the two closest, we only have to recompute the distances to the new cluster. We stop the clustering algorithm when the distance between two clusters exceeds a certain threshold $\sigma \in\left[0, \frac{\pi}{2}\right]$.

Hierarchical clustering algorithms have complexity of $O\left(n^{2}\right)$ with $n$ the number of elements to be clustered. In our case, the number of elements is $k *(k-1)$. So in total, our clustering has complexity of $O\left(k^{4}\right)$. The complexity of the clustering thus strongly depends on the size $k$ of the neighborhoods. Since we use not very huge $k$ in our method (normally $k=16$, at most $k=32$ ), the method overall is still reasonably fast.

Analysis. All clusters containing only a few points are discarded, since they correspond to the noisy points. The remaining clusters are analyzed as follows. Opposite clusters on the sphere are considered as one cluster. If in the end a single cluster remains we decide that the current point does not belong to a feature. If two, three or four clusters remain, we decide that the point belongs to a sharp feature. If more than four clusters remain, we decide that the current point does not belong to a feature. The number of four seems to be a good value, since most data sets generally don't have more than four sharp features meeting in one point, see Figure 5 for examples. Even the more complex vase in Figure 16 does not have sharp feature corners with more than three edges. However, it can be adapted in presence of a particular data set if necessary. Let us now discuss in more detail, how to choose the parameters.

\section{LOCAL-ADAPTIVE METHOD}

The decision, to declare a sample point as sharp feature or not, is depending on the size of the local neighborhood $(k)$ and the sensitivity parameter $(\sigma)$.

\subsection{Size of neighborhood}

The size of the neighborhood is user controlled and fixed. It represents the region tested for a feature and it influences the computation time.

On one hand, a too small neighborhood will not deliver enough information for a reliable result. On the other hand, a too big neighborhood represents a huge region and thus even features far away from the sample point would influence the result. This may lead to false positives, i.e finding sharp features in flat regions. A too big neighborhood may also violate the requirement that all points of the neighborhood belong to the same connected region of the underlying surface. This is a theoretical problem, which does not occur with the relatively small number of neighbors we choose. However, this problem has been pointed out by Pauly et al. [PKG03], since their algorithm loops on the size of the neighborhood varying from 1 to 200 . A criterion is proposed to detect invalid neighborhood sizes.

So there is a trade off needed for the size of the neighborhood. During our tests a neighborhood with a size of $k=16$ performs best. Detection methods for general features come to a very similar conclusion. [GWM01] propose $k=10$ to 16. [WW07] propose $k=12$. Sizes of 8 and 32 also deliver acceptable results but were kind of lower and upper bounds.

\subsection{Sensitivity parameter for Gauss map clustering}

Remember that during clustering the distance $D_{c}$ (4) between two clusters is compared to a threshold value. This threshold value is the sensitivity parameter $\sigma$. The clustering algorithm stops merging the clusters, when the distance between the clusters exceeds the threshold. $\sigma$ corresponds therefore the minimal distance between all resulting clusters. 

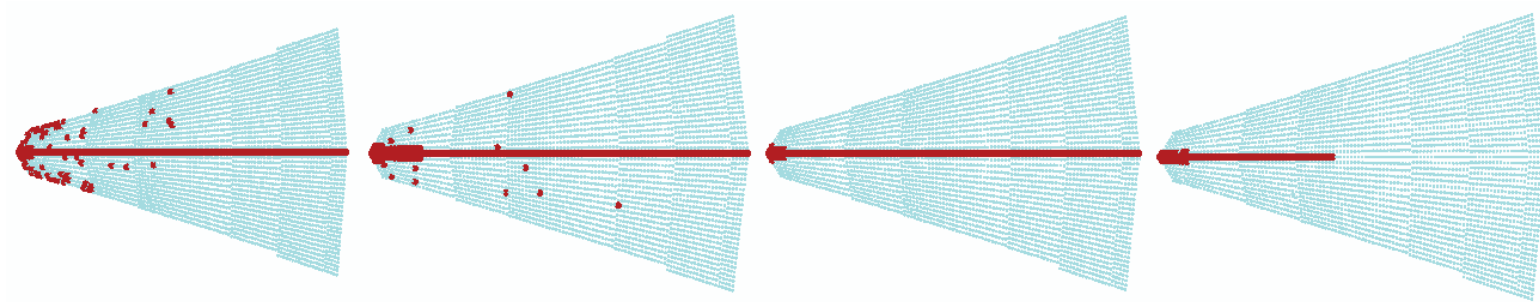

Fig. 6. Feature detection with a global sensitivity value $\sigma$. The points are sampled on a piecewise bilinear surface with a sharp feature line, similar to the example in Fig.7-left. The angle between the surfaces varies along the feature line from acute $\left(45^{\circ}\right)$ to obtuse $\left(140^{\circ}\right)$. The detected feature points are highlighted by fat red points. $\sigma=0.05,0.1,0.6,1.0$ left to right.

Let us explain the role of this parameter by first recalling the two main requirements to our method:

1) detection of all points lying on a sharp feature

2) no selection of points which are close to the feature, but not on it.

These requirements will lead our method to detect a relatively sparse set of points lying on the sharp feature or very close to it. Note that the second requirement is particular to our method, since in most cases, a sharp feature corresponds to a line. All previous methods are different in the sense that they aim to detect general features (not sharp features). Such a feature often corresponds to surface region with high curvature variation. Consequently, they compute many points on the feature followed by a post-processing step, which reduces the number of points and which replaces them by an approximating line [GWM01], [PKG03].

The value fixed for the parameter $\sigma \in\left[0, \frac{\pi}{2}\right]$ makes the method more or less sensitive for sharp feature detection. The sensitivity is inverse reciprocal to the value of $\sigma$. Let us first investigate theoretically how the method is expected to behave for big and small sensitivity values, before studying a numerical example: A big value of $\sigma$ corresponds to clusters with a big distance between them obviously implies that clusters would consist of many points. This would work well for features which are distinguishly very sharp, i.e. features with a right angle or an acute angle. But at the same time it would ignore features with an obtuse angle. In this case it is more difficult to distinguish the noisy points from the correct surface normals. If $\sigma$ is too big, one would end with only one cluster containing all points of the Gauss map and the sample point would not be recognizes as a feature, violating requirement 1 . A small value of $\sigma$ stops clustering earlier. It would result in clusters which are more close together corresponding to feature with an obtuse or an acute angle. One one hand the method becomes thus more sensitive for detection of critical sharp features. On the other hand it may then be difficult to distinguish feature points from neighbor points, since their clustering behavior is very similar. It would violate requirement 2 .

It seems that there might be some critical features which are difficult to detect. In practice, the choice of a

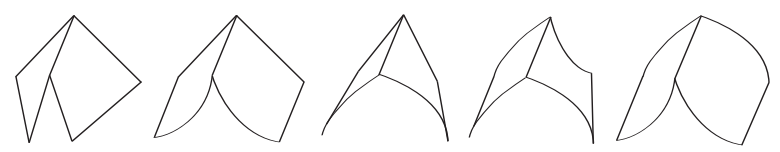

Fig. 7. Some examples of sharp feature profiles with varying angles.

user-controlled global sensitivity parameter works well for many examples. But for more complex examples, a global sensitivity parameter does not show optimal results in presence of acute and flat angles in the same data set. We already suspected this behavior in the previous paragraph, so let us study now the limits of the present method with a particular numerical example. We constructed a piecewise bilinear surface with a straight feature line where the angle between the surface varies from acute $\left(45^{\circ}\right)$ to obtuse $\left(140^{\circ}\right)$. This data set corresponds to the left example in Figure 7. In Figure 6 is shown the result of feature detection for $\sigma=0.05,0.1,0.6,1.0$. It can be observed, that a very small value of $\sigma=0.05$ or 0.1 produces many overrates not only near acute features, i.e. points which are falsely detected. A mean value of 0.6 detects all features but still produces overrates near acute features. A big value of $\sigma=1.0$ however fails to detect flat feature points.

Obviously, if the point cloud contains features with acute angles and features with obtuse angles, one global parameter for sensitivity is not sufficient. A good global value for obtuse angles allows to detect all features, but will probably overrate features in regions of acute angles. In this case a whole region might get marked as sharp feature while the exact position of the feature cannot be determined. So a global value always needs a trade-off between finding all features (requirement 1) and finding the exact position of the features (requirement 2).

This observation motivated us to develop an extension of the algorithm which is presented in the next section.

\subsection{Adaptive and local sensitivity parameter}

In the previous section it has been demonstrated, that the use of a global sensitivity parameter $\sigma$ might not be sufficient in all cases. In the present section we will show how to make the parameter $\sigma$ local and adaptive. The aim is to develop a method that changes the sensitivity 

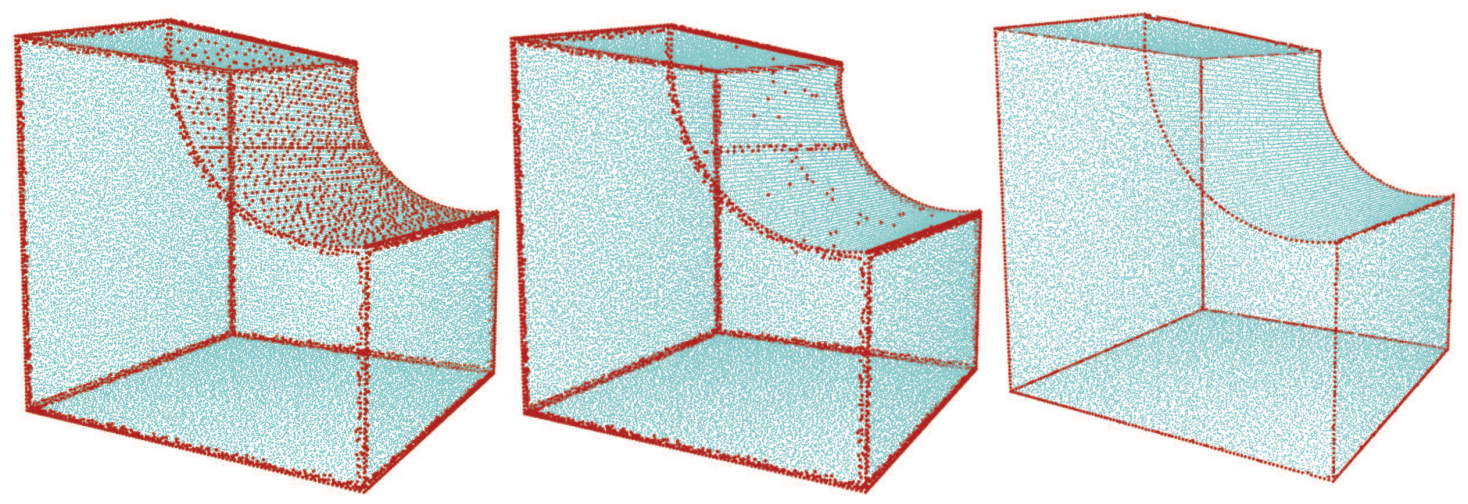

Fig. 8. The two pictures on the left show a non-uniformly sampled point cloud with an overrated set of feature points obtained with two different parameter settings $\sigma=0.1, k=20$ (left), $\sigma=0.5, k=16$ (middle). The right figure is the result obtained with the iterative method applied to the overrated examples. $\sigma$ and $k$ are automatically adapted for each of the feature candidates.

value adaptively for different regions of the point cloud. It should compute automatically an optimal $\sigma$ value for each feature candidate. Furthermore we also adapt the neighborhood size $k$ automatically, so that the user is no longer obliged to adjust the two parameters manually in order to get good results.

We achieve this by an iterative process. In a first initialization step the method described in Section 3 including flatness test on the whole point set and Gauss map clustering on the selected points does a feature search using global values for $\sigma$ and $k$. In this first passage, $\sigma$ will be set to a relatively low value while $k$ will be relatively large. This delivers a set of many feature candidates. We generate a larger set in order to not miss any feature points with an obtuse angle, at the cost of also getting overrated acute angle features (see Figure 8). Since we now have a candidate list with many outliers, we reduce the number of candidates in the following iteration steps. In these steps the method checks the number of possible features in the neighborhoods of the feature candidates.

In the case of a sharp feature e.g. an edge or a curved line, the feature points will lie on a line dividing the neighborhood in two parts. The percentage of feature candidates inside this neighborhood will be relatively low. A very high percentage of feature candidates inside a neighborhood indicates that the sensitivity was too high, i.e. value of $\sigma$ too low, for this neighborhood. The existence of only one or no other feature candidate in the neighborhood indicates that the candidate is not a sharp feature but an outlier. Thus increasing carefully the sensitivity value $\sigma$ would reduce the number of overrated points, see Figures 6 , and 10. To also get rid of the overrated features at acute angles which don't disappear with increasing $\sigma$, we also locally reduce the size of the neighborhoods at every iteration step.

The algorithm for each iteration step is the following. We raise $\sigma$ by $10 \%$ in the neighborhood of the current candidate and reduce the neighborhood size $k$ by one.

Then only the candidate features inside this neighborhood are tested again for being a sharp feature using the Gauss map clustering described above. The raised sensitivity and the smaller neighborhood size lead to a reduction of the number of candidates in this neighborhood.

This step iterates until either the percentage of features in the neighborhood of the candidate reaches a reasonable value (during our experiments a value of $30 \%$ of neighborhood size worked well) or another break condition is reached. These additional breaking conditions can be chosen among maximum value for the sensitivity $(\sigma=1.2)$, a minimal size for the neighborhood $(k=8)$ and a maximum number of iteration steps.

The iteration process in pseudocode:

while ( iteration break criteria not reached ) \{

for ( Candidate in Feature Candidates ) \{ initParameters( $\mathrm{n}$, sigma )

Neighborhood $=$ Candidate. Neighborhood

while (Neighborhood.getNumberOfFeatureCandidates() $\geq$ threshold ) \{

adjustParameters( $n$, sigma )

//Check Candidate

isFeature $=$ checkforFeature $($ Candidate, $n$, sigma $)$

if $($ isFeature $==$ false $)$

removeFromCandidateList( Candidate )

// Check Neighborhood of Candidate for ( Neighbor $\in$ Neighborhood )

if ( Neighbor.isFeature() ) \{

isFeature $=$ checkforFeature ( Neighbor, n, sigma )

if $($ isFeature $==$ false $)$ \} removeFromCandidateList( Neighbor)

\}

\}

The right picture in Figure 8 is the result obtained with the iterative method applied to the overrated 
examples. $\sigma$ and $k$ are automatically adapted for each of the feature candidates. The two pictures on the left show a point cloud with an overrated set of feature points obtained with two different parameter settings $\sigma=0.1, k=20$ (left), and $\sigma=0.5, k=16$ (middle).

Adding iterative refinement steps to a process often increases significantly computational costs. Especially regarding large point clouds, checking the neighborhood of every data point iteratively is very costly, $O(N k)$ in the worst case where each point has been selected as a feature candidate. This scenario is of course unrealistic.

In the present setting, the additional computational costs resulting from the iteration steps are quite low for two reasons. First, the number of data points checked during the first iteration is negligible with respect to the initial data set. They correspond to the feature candidates selected after the first pass using the Gauss map clustering with a set of not optimal global parameters. In the case of the "cube with hole" examples, they present only $11 \%$ of the point cloud. In the case of the cube without the corner, they present $9 \%$. Second, the number of remaining feature candidates decreases significantly after each iteration. This results from the fact, that each iteration of a single neighborhood will also eliminate a whole group of other candidates inside this neighborhood, since the neighborhoods of close points intersect each other. That means that the neighbors of the candidate, in case of a being not a sharp feature, are likely to be eliminated from the candidate list before their their own neighborhood has been tested. This reduces the number of candidates very fast and fewer neighborhoods in the cloud will be tested again. In a usual scenario after the first step only a small percentage around $10 \%$ or less of the point cloud will be marked as candidate for a sharp feature. This makes the additional computational costs for the refinement iterations significantly lower than the costs for the first global feature search.

\section{RESUlts}

We have implemented the sharp feature detection pipeline described in the previous sections. We use both points sets sampled from some known geometries, such as the cubes and the bilinear surfaces, as well as more complex models, such as the "fandisk" the "vase", and the "trim-star". To all models we applied both the basic algorithm described in Section 3 where we tried to find some optimal global parameters by testing many combinations, and the improved local-adaptive method with an automatic and adaptive choice of the optimal local parameters. The robustness of the method is tested and evaluated with respect to two aspects: the variation of the angle of a sharp feature and noise.

\section{Robustness w/r to varying angles}

Let us first test the robustness of the method with

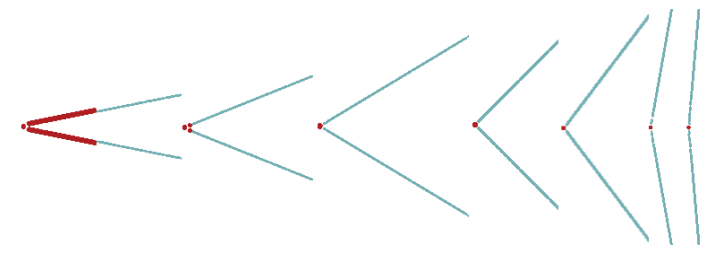

Fig. 9. Feature detection on different angles using the local adaptive method.

regard to very acute and obtuse angles by testing two planes connected with a fixed angle between them. The profiles of these surfaces are shown in Figure 9. ¿From left to right the angles are $25^{\circ}, 45^{\circ}, 60^{\circ}$, $90^{\circ}, 110^{\circ}, 140^{\circ}, 160^{\circ}$ and $170^{\circ}$. The method works perfectly for all angles greater then $45^{\circ}$, even very obtuse angles don't cause any problem. The method also allows angles of $45^{\circ}$ and less, but with very acute angles the results are no longer very precise. In this case, the overrated points don't disappear even when increasing $\sigma$ and decreasing $k$. For the three acute angles the neighborhood was $k=8$ for all others we chose $k=10$. The problem of not detected features appears only with very obtuse angles near $180^{\circ}$, which is negligible.

\section{Comparison between global and local-adaptive method}

We then compare the performance of both methods (global and local-adaptive) using three test examples with a known number of sharp feature points. The first example is a simple cube with 580 sharp feature points. The second example is a surface containing a sharp edge with a varying angle having 256 sharp feature points. At one end the angle is $\left(45^{\circ}\right)$ at the other end it is $\left(140^{\circ}\right)$. The third example is cube with a hole with 1350 sharp feature points. We checked the global method with different sets of parameters and compare it to the localadaptive method.

\section{Global method}

Figure 10 shows the results for the global method. For each example 9 sets of parameters have been tested. The three lines correspond to $k=10,16,20$, the three columns correspond to $\sigma=0.1,0.5,0.8$.

The simple cube example shows, that for a dataset with only one kind of angle it is possible to find good global values for $\sigma$ and $k . k=10$ gives best results, see Figure 10 (first line). The optimal value of $\sigma=0.6$ was found by trying several times the algorithm. It not fits into the figure here, but it results in a perfect feature detection of all 256 points of the cube.

In the example of two surfaces joining with the varying angle, one can see the problems of the global method. A global value of $\sigma$ is not sufficient. On one hand, the feature points on the right part of the edge, where the angle become obtuse, are not detected when $\sigma=0.8$ is big (right column) in Figure 10. On the other hand, 

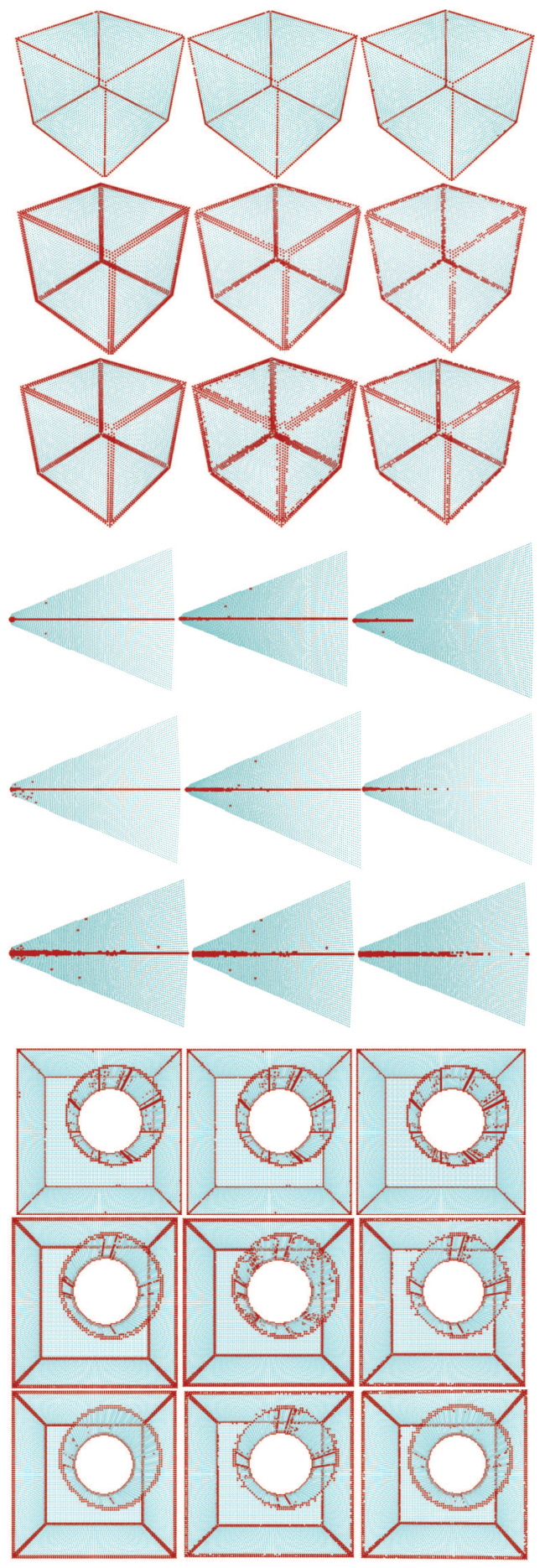

Fig. 10. the examples used for the study with the global method. For each example 9 sets of parameters have been tested. The three lines correspond to $k=10,16,20$, the three columns correspond to $\sigma=0.1,0.5,0.8$.

a small $\sigma$ can detect them, but leads to outliers and overrates in acute angle regions ${ }^{1}$. The size of the neighborhood $k$ has a huge influence on the method too.

The importance of $k$ is best seen in the cube-with-hole

\footnotetext{
${ }^{1}$ The pdf file allows a zoom-in, in order to see all Figures with more precision.
}

example. On one hand, a small neighborhood $k=10$ performs best on the outside edges of the cube, but leads to bad results in the curved region inside the hole. On the other hand, a big neighborhood $k=20$ performs better in the curved areas, but worse on the outside edges of the cube.

All these examples confirm the conclusion we already did in Section 3: a global choice of the parameters can not detect all features satisfactory.

\section{Local-adaptive method}

The local-adaptive method can take a huge advantage on its capability to vary $\sigma$ and $k$. For each feature candidate detected in a first pass, an optimal value of $\sigma$ and $k$ is determined and outliers are eliminated. However, the method is not able to add feature points during the iterations, since the final set of detected features is a subset of the feature candidates. Therefore, one has to initialize the iteration with a set of parameters, which won't neglect possible features. The experiences in the previous paragraph show that $\sigma=0.1$ and $k=20$ are good initial values. The result of feature point detection using the local-adaptive method is shown in Figure 11. Table 1 resumes the performance of the algorithm with respect to the three hand-made examples.
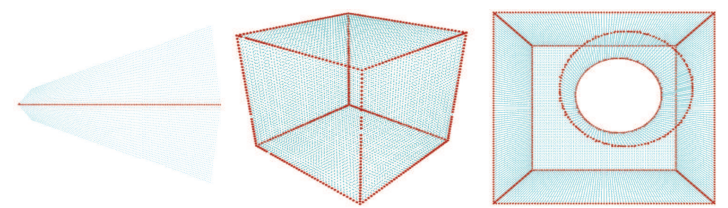

Fig. 11. feature detection using the local-adaptive method.

\begin{tabular}{|l|c|c|c|c|c|}
\hline & \# points & $\begin{array}{c}\text { feature } \\
\text { points }\end{array}$ & detected & $\%$ & outliers \\
\hline cube & 10250 & 580 & 575 & $99 \%$ & \\
\hline planes & 6090 & 256 & 257 & $100 \%$ & 1 \\
\hline hole & 58720 & 1350 & 1398 & $100 \%$ & 48 \\
\hline
\end{tabular}

TABLE 1

TEST RESULTS FOR THE LOCAL-ADAPTIVE METHOD.

\section{Robustness to noise}

In order to test and quantify the sensitivity of our local adaptive method to noise, we compare distances measured between real feature points on a test point cloud and on a perturbed point cloud. The test example is the cube-with-hole, since it has corner, convex and concave feature points. All points are lying in a ball of radius $R=8.5$. The original point cloud has 17400 uniformly sampled points. Distance between neighboring points is 0.25 . The perturbed point cloud is obtained by adding to each point a random vector chosen in a ball whose size is $0.4 \%, 0.8 \%$ and $1.2 \%$ of $R$.

The error induced by the noise is measured using the distances between the set of feature points in the original 


\begin{tabular}{|c|c|c|cc|}
\hline Noise & $\delta_{\infty}$ & $\delta_{\text {avg }}$ & \multicolumn{2}{|c|}{ \# features } \\
\hline $0.0 \%$ & 0 & 0 & 839 & $(+0)$ \\
\hline $0.4 \%$ & 0.25 & 0.02 & 862 & $(+23)$ \\
\hline $0.8 \%$ & 0.26 & 0.03 & 875 & $(+36)$ \\
\hline $1.2 \%$ & 0.31 & 0.08 & 984 & $(+145)$ \\
\hline $1.6 \%$ & 0.75 & 0.19 & 1245 & $(+406)$ \\
\hline $2.0 \%$ & 0.93 & 0.21 & 1255 & $(+416)$ \\
\hline
\end{tabular}

TABLE 2

DISTANCES BETWEEN ESTIMATED FEATURES AND REAL FEATURES OF THE CUBE-WITH-HOLE MODEL. NOISE AS A PERCENT OF THE MODEL RADIUS VARIES. THE NUMBER OF EXACT FEATURES 839 IS COMPARED TO THE NUMBER OF ESTIMATED FEATURES.

point cloud $Q=\left\{\boldsymbol{q}_{i}\right\}$ and the set of estimated feature points in the perturbed point cloud $P=\left\{\boldsymbol{p}_{i}\right\}$. The distances $\delta_{\infty}$ and $\delta_{a v g}$ are defined similar to [MOG09]. $\delta_{\infty}$ is the maximal distance between an estimated feature point $\boldsymbol{p}_{i}$ and its nearest feature point in $Q . \delta_{\text {avg }}$ is the average distance between an estimated feature point $\boldsymbol{p}_{i}$ and its nearest neighbor in $Q$.

Table 2 summarizes the results of the experiment for different noise radii. The number of detected feature points is also a measure of quality, since our method aims to detect only feature points or a sparse set of points very close to a feature,

$\delta_{\infty}$ measures the presence of outliers. The experiment shows that in the case of mean noise $(0.8 \%)$ the distance of isolated outliers $\delta_{\infty}=0.26$ is equal to the point distance $(0.25)$ in the original point cloud. Outliers are thus very close the neighbors of real features, see Figure 12. For bigger noise of $1.2 \%$ the isolated outliers are still very close to the feature points, but their number increases. Even though the number of so-called false features increases with increasing noise, the detection method is still stable, since isolated outliers stay close to real features, even for big noise.
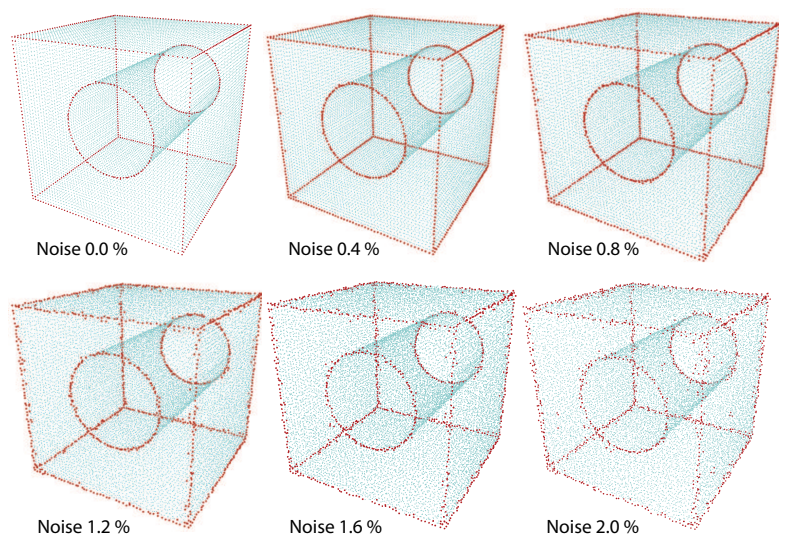

Fig. 12. Estimated feature points on noisy point clouds. The original cube-with-hole model is perturbed with random noise.

\section{Complex point-sampled surfaces}

After these self constructed examples let us show some more real world examples. Here it is not possible anymore to measure the quality of the output of the
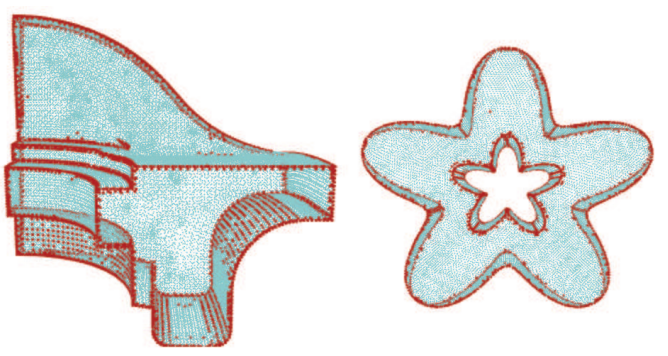

Fig. 13. The global method on the fandisk and the trim-star.

algorithm, since the feature points are not known and generally don't ly directly on the feature as stated in [AFRS05]. Only a visual control is possible. First we tried the global method on the well-known fandisk $(\sigma=0.7, k=12)$ and the trim-star $(\sigma=0.6, k=12)$. It can be seen in Figure 13 that no optimal result can be achieved. We then applied the local-adaptive method to the following three examples. Figure 14 shows the detected sharp feature points on the fandisk model (41250 vertices). The local method here again delivers good results. The next examples in Figures 15 and Figure 16 show the trim-star model (25100 vertices) and the vase ( 896000 vertices) which has some curvy, sharp features. Notice, that for the vase and the trimstar, the original model is a triangulation, but the points are NOT lying on the feature. The triangle edges are zig-zagging along the feature lines.
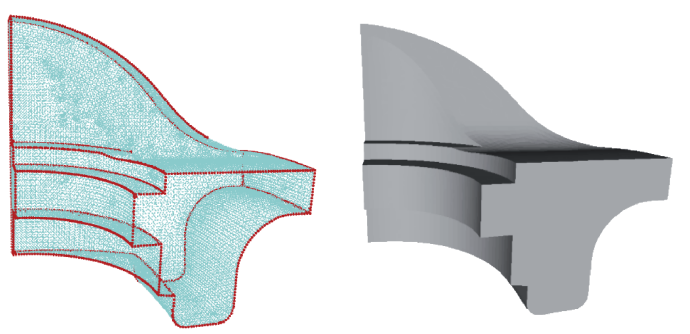

Fig. 14. Sharp feature detection on the fandisk model.
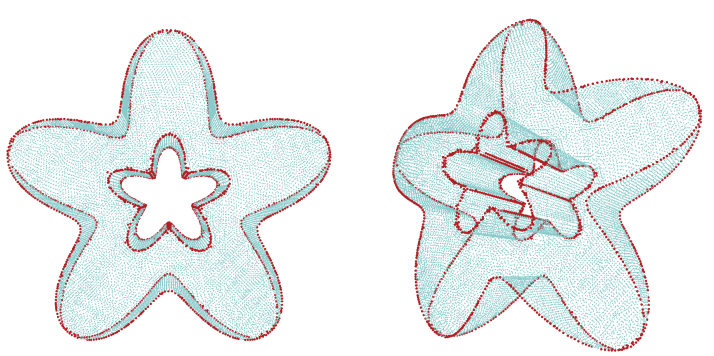

Fig. 15. Sharp feature detection on the trim-star model.

Finally we used a scan data set by a Cyberware ${ }^{T M}$ scanner of a drill. Many data is missing near the sharp features and the precision is quite rough, see Figure 17, but the feature lines are correctly detected along the 
sharp lines of the drill.
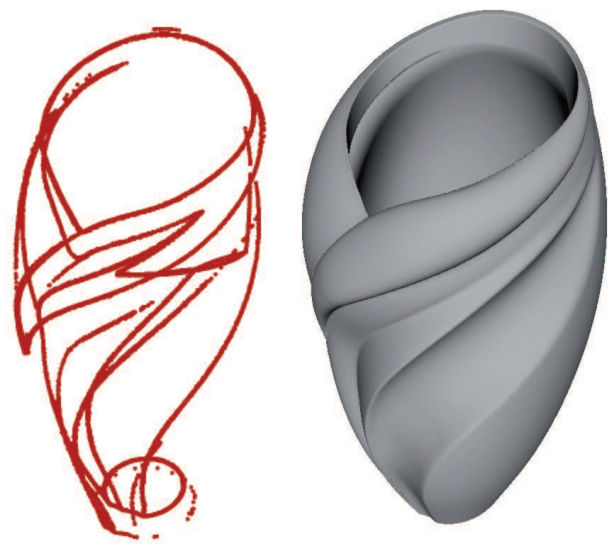

Fig. 16. Sharp feature detection on the vase model.

Concerning computation time, our local-adaptive algorithm runs for the fandisk example with 41520 vertices in about 19 seconds, for the trim-star with 24444 vertices in $30 \mathrm{~s}$ and for the drill with 23994 vertices 40 seconds. 0.6 seconds pre-processing time costs the knearest computation for the fandisk (PC: Xeon 3.0Ghz). The vase model takes $20 \mathrm{mn}$ because of the time consuming and repeated clustering in the local method. This is however reasonably fast for most available models up to $100 \mathrm{k}$ points, and compares well to the multiscale PCA method, even though our implementation is not done optimally.

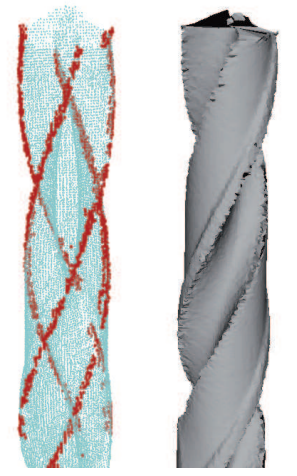

Fig. 17. Sharp feature detection on a drill scan by a Cyberware ${ }^{T M}$ scanner. The data set is very rough and has missing data near the sharp feature.

\section{Comparison to PCA methods}

The ability of our method to detect sharp feature points is the key characteristic and can be seen as a supplement to all previous methods. In fact, the notion of "sharp" is important here. Previous methods, including [GWM01], [PKG03], [MOG09], aim to detect more general features in unstructured point clouds such as high curvature or curvature variation regions. These methods result in large bands of feature points, even in the presence of sharp features. A post-processing step is then proposed in [GWM01], [PKG03] to sparsen this set of points followed by an approximation with a smooth curve. All methods use variants of a principal component analysis (PCA) of the neighborhood of a point in order to estimate local curvature values.

In order to compare our local Gauss map clustering to PCA based feature detection for the special case of sharp features, we implemented the PCA part of the multiscale feature detection method [PKG03]. It is a powerful method and easy to implement. Without measuring exactly the differences, Figure 18 makes a visual comparison. As it has been expected, by tuning several times the parameters of the multiscale method (surface variation 0.08 , neighborhood size varies from 10 to 40 , feature weight with lower and upper border 25 and 30, see [PKG03]), we always end up with a wide band of estimated feature points which call for a postprocessing sparsening in contrast to local-adaptive Gauss map clustering. It confirms that PCA-based methods are not best appropriate for sharp feature detection.
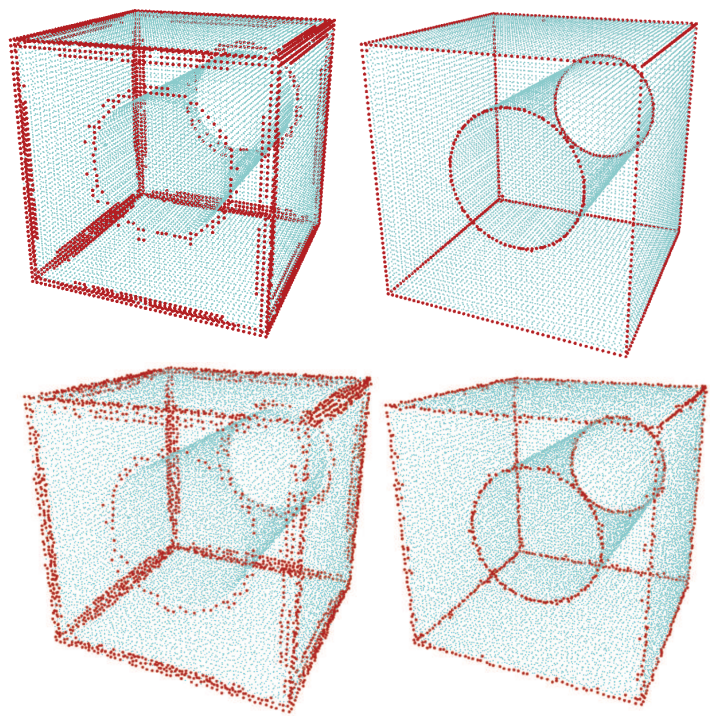

Fig. 18. Comparison of PCA-based feature detection (left) with our local-adaptive Gauss map clustering method (right). Both methods are applied to the original uniformly sampled point cloud of the cube-withhole example (upper row) and to the perturbed data set with $1.2 \%$ of noise (lower row).

\section{CONCLUSION AND FUTURE WORK}

We have presented a new method for sharp feature detection on point-sampled geometry. The proposed method uses Gauss map clustering for feature detection. It is fully automatic, without any user interaction. It does not rely on local surface reconstructions, and no normal information is required. A key contribution is the integration of an adaptive local sensitivity parameter for the feature identification, which reduces the user dependency of the method. Many tests have been performed in order to demonstrate that the method works 
very successfully even on very complex geometry in contrast to algorithms based on global parameters. The resulting point cloud with the marked sharp features can be used for several applications now (surface reconstruction, non-photorealistic rendering, mesh generation, MLS-surface modeling). In [GWM01] a hole section is devoted to possible applications. The properties of the resulting feature point set (preciseness, sparseness, very few outliers) make the method an excellent preprocessing step for feature line approximation with smooth spline curves [GWM01].

All line-type, corner-type sharp features are detected. Cone peaks are not treated here, the method needs to be adapted in order to recognize the particular clustering behavior in this case.

\section{ACKNOWLEDGMENTS}

The vase model is provided courtesy of INRIA, the fandisk and trim-star are provided courtesy of MPII, all by the AIM@SHAPE Shape Repository. The drill model is courtesy of Sergei Azernikov, Siemens Corporate Research, Princeton.

This work was supported by the IRTG 1131 of the DFG (German Research Foundation). It has been partially supported by the Deutsche Forschungsgemeinschaft INST 248/72-1.

\section{REFERENCES}

[ACK01] Amenta N., ChOI S., Kolluri R. K.: The power crust. In SMA '01: Proceedings of the sixth ACM symposium on Solid modeling and applications (New York, NY, USA, 2001), ACM, pp. 249-266.

[AFRS05] AtTene M., Falcidieno B., Rossignac J., SpagnUOLO M.: Sharpen\&bend: Recovering curved sharp edges in triangle meshes produced by feature-insensitive sampling. IEEE Transactions on Visualization and Computer Graphics 11, 2 (2005), 181-192.

[AGJ00] AdAmy U., Giesen J., John M.: New techniques for topologically correct surface reconstruction. In VISUAL IZATION '00: Proceedings of the 11th IEEE Visualization 2000 Conference (VIS 2000) (Washington, DC, USA, 2000), IEEE Computer Society.

[AMN*98] Arya S., Mount D. M., Netanyahu N. S., SilVERMAN R., WU A. Y.: An optimal algorithm for approximate nearest neighbor searching fixed dimensions. J. ACM 45, 6 (1998), 891-923.

[BL97] BeIS J. S., LOWE D. G.: Shape indexing using approximate nearest-neighbour search in high-dimensional spaces. In CVPR '97: Proceedings of the 1997 Conference on Computer Vision and Pattern Recognition (CVPR '97) (Washington, DC, USA, 1997), IEEE Computer Society, p. 1000 .

[CLR01] CORMEN T. H., LEISERSON C. E., Rivest R. L.: Introduction to Algorithms, 2th ed. MIT Press and McGrawHill, Boston, MA, USA, 2001.

[DOHS08] Daniels J., Ochotta T., Ha L. K., Silva C. T. Spline-based feature curves from point-sampled geometry. Vis. Comput. 24, 6 (2008), 449-462.

[DVVR07] Demarsin K., Vanderstraeten D., Volodine T., Roose D.: Detection of closed sharp edges in point clouds using normal estimation and graph theory. Comput. Aided Des. 39, 4 (2007), 276-283.

[FCOS05] Fleischmann S., Cohen-Or D., Silva C.: Robust moving least-squares fitting with sharp features. $A C M$ Trans. Graph. (2005), 37-49.
[GM97] GUY G., MEdioni G.: Inference of surfaces, 3d curves, and junctions from sparse, noisy,3d data. IEEE Transactions on Pattern Analysis and Machine Intelligence 19, 11 (1997), 1265-1277.

[GWM01] Gumhold S., WANG X., McLeod R.: Feature extraction from point clouds. Proceedings of 10th International Meshing Roundtable (2001).

[HDD*92] Hoppe H., DeRose T., Duchamp T., McDonald J., STUETZLE W.: Surface reconstruction from unorganized points. In SIGGRAPH '92: Proceedings of the 19th annual conference on Computer graphics and interactive techniques (New York, NY, USA, 1992), ACM, pp. 7178.

[HG01] Hubeli A., Gross M. Multiresolution feature extraction for unstructured meshes. Proccedings of IEEE Visualization (2001), 287-294.

[HPW05] Hildebrand K., Polthier K., WardetzKy M. Smooth feature lines on surface meshes. Proceedings of Symposium on Geometric Processing (2005).

[HTF09] Hastie T., TibshiRANi R., FriedMan J.: The Elements of Statistical Learning: Data Mining, Inference, and Prediction., 2th ed. Springer, Boston, MA, USA, 2009.

[JPR00] Junghyun H., PRATt M., Regli W.: Manufacturing feature recognition from solid models: a status report. IEEE Transactions on Robotics and Automation 16, 6 (2000), 782-796.

[KBSS01] Kobbelt L., Botsch M., Schwanecke U., Seidel H.-P.: Feature sensitive surface extraction from volume data. In SIGGRAPH '01: Proceedings of the 28th annual conference on Computer graphics and interactive techniques (New York, NY, USA, 2001), ACM, pp. 57-66.

[LW77] LEE D., WONG C.: Worst-case analysis for region and partial region searches in multidimensional binary search trees and balanced quad trees. Acta Informatica 9 (1977).

[LX08] LIU Y., XIONG Y.: Automatic segmentation of unorganized noisy point clouds based on the gaussian map. Comput. Aided Des. 40, 5 (2008), 576-594.

[MOG09] Mrigot Q., OvSJANikov M., Guibas L. J.: Robust voronoi-based curvature and feature estimation. In Symposium on Solid and Physical Modeling (2009), Bronsvoort W. F., Gonsor D., Regli W. C., Grandine T. A., Vandenbrande J. H., Gravesen J., Keyser J., (Eds.), ACM, pp. $1-12$.

[OGG09] Oztireli C., Guennebaud G., Gross M.: Feature preserving point set surfaces based on non-linear kernel regression. Computer Graphics Forum 28, 2 (2009).

[PKG03] PAUly M., KeISER R., Gross M.: Multi-scale feature extraction on point-sampled surfaces. Computer Graphics Forum (2003).

[WB01] Watanabe K., Belyaev A. G.: Detection of salient curvature features on polygonal surfaces. Computer Graphics Forum (2001), 385-392.

[WG09] Weinkauf T., GNTHER D.: Separatrix persistence: Extraction of salient edges on surfaces using topological methods. Computer Graphics Forum (Proc. SGP '09) 28, 5 (July 2009), 1519-1528.

[WW07] WU J., WANG Q.: Feature point detection from point cloud based on repeatability rate and local entropy. In Proceedings of the SPIE Vol.6786 (2007), p. 67865. 